\title{
BILATERAL OVARIAN NEOPLASM IN ADOLESCENT GIRL: A RARE ENTITY
}

N. Gopal, Prashant S. Joshi, Vinayachandra Singh.

1. Professor. Department of Obstetrics \& Gynaecology, Adichunchanagiri Institute of Medical Sciences,

2. Associate Professor. Department of Obstetrics \& Gynaecology, Adichunchanagiri Institute of Medical Sciences,

3. Post Graduate Resident. Department of Obstetrics \& Gynaecology, Adichunchanagiri Institute of Medical Sciences,

\section{CORRESPONDING AUTHOR:}

Dr. Prashanth S. Joshi,

Flat No. 201, Nanar Sorrento,

$36^{\text {th }}$ main, $3^{\text {rd }}$ Cross, BJM I Stage,

Madivala Dollars Colony, Bangalore-560068.

E-mail: joshpj@rediffmail.com.

ABSTRACT: A 20 year old girl presented with mass per abdomen and failure to attain menarche. She had undergone previously left salpingo-oophorectomy surgery for torsion of left ovary, histopathology of which showed dysgerminoma of left ovary. After a thorough investigation, she underwent staging laparotomy followed by hysterectomy with right salpingooophorectomy for stage $1 \mathrm{C}$ dysgerminoma

KEY WORDS: Dysgerminoma, Torsion, Salpingo-oophorectomy

INTRODUCTION: Dysgerminomas are the most common malignant germ cell ovarian carcinoma. Most dysgerminomas occur in adolescence and early adult life; 5\% occur in prepubertal children, and they are extremely rare after 50 years of age1. Abnormal gonads (due to gonadal dysgenesis and androgen insensitivity syndrome) have a high risk of developing a dysgerminoma. Most dysgerminomas are associated with elevated serum Lactate Dehydrogenase (LDH), which is sometimes used as a tumour marker. Dysgerminomas present as bilateral tumours in $10 \%$ of patients and in a further $10 \%$ there is microscopic tumour in the other ovary. Dysgerminomas can be located in the brain, usually arising in the hypothalamic or epiphysial regions.

CASE REPORT: A 20 year old presented with failure to attain menarche, Pain abdomen since 2 months, Mass per abdomen since 2 months, Mild dull aching diffuse lower abdominal pain.

She gives history of having an abdominal surgery at the age of 13 yrs in a private hospital for acute pain abdomen and vomiting. As per hospital records, she underwent diagnostic laparoscopy followed by laparotomy which showed torsion of left ovarian tumour and she underwent left salpingo-oophorectomy. The histopathology showed dysgerminoma of left ovary.

On examination, she was pale and ill looking with no significant lymphadenopathy, no acne, Breasts-Tanner stage I, nipples not widely spaced \& no galactorrhoea, no hirsutism and no webbed neck. External Genitalia examination revealed that pubic hair was Tanner stage I, labia majora and minora appeared normal (Figure1). Vagina admitted one finger with difficulty and Cervix was not felt. Routine blood \& Biochemistry investigations were normal. Serum marker was negative for HCG and Positive for LDH. Buccal smear was Positive for Barr body ( $>18 \%$ ), 
Karyotyping was 45X0. Abdominal Ultrasound showed that uterus was compressed due to right sided adnexal mass extending from pelvis to epigastrium. Mass was solid lobulated with cystic components in the centre, showing septations and loculations (Figure2). Bilateral hydroureteronephrosis was present .CT scan revealed a well defined soft-tissue-density mass lesion of 20x12x10 cm occupying predominantly right iliac region with superior extension up to right hypochondriac region medially crossing the midline. Few areas of cystic hypo-dense and necrotic areas noted within the mass (Figure3). There was mass effect on bilateral ureters causing mild bilateral hydroureteronephrosis. There was no evidence of paraaortic, retroperitoneal or pelvic lymphadenopathy, and ascites. Both ovaries could not be visualized. Uterus was hypoplastic $2 \times 3 \times 2 \mathrm{~cm}$. There was no evidence of intraperitoneal/retroperitoneal/ solid organ metastasis. Mass appears to displace bowel loops to left abdominal quadrants.

TREATMENT: Staging laparotomy was done followed by TAH with right salpingooophorectomy. Per operatively right ovarian mass of $20 \times 15 \times 10 \mathrm{~cm}$ with bosselated surface with intact capsule was found(Figure4). Uterus appeared infantile. Under surface of the diaphragm, liver, omentum were free of metastatic growth. Para aortic nodes were not enlarged. Peritoneal fluid sample and omental biopsy were taken. Final Diagnosis was Stage I C Dysgerminoma.

On histopathological examination, macroscopic appearance showed Irregular grey white lobulated mass of 20x12x11 cm. External surface showed stretched out fallopian tube measuring $11 \mathrm{~cm}$. On Cross Section, grey white lobulated homogenous and yellowish solid solitary cyst with areas of necrosis. Microscopic appearance showed monomorphic cellular tumour composed of round to polygonal cells with large vesicular and prominent nucleoli. Cells arranged in sheets, cords, pseudoalveolar formation and peritheliomatous pattern. Fibrovascular septae were seen separating sheets and groups of cells with lymphocytic infiltration (Figure5). Capsule showed partial thickness infiltration. Mitotic activity was not significant. Peritoneal fluid analysis was positive for malignant cells. Omental biopsy was normal.

DISCUSSION: Dysgerminoma represents the ovarian counterpart of testicular seminoma. These two tumors are histologically identical and the term germinoma has been proposed for both. It usually occurs in normally developed females but is the most frequent ovarian malignant tumour found in dysgenetic females, testicular feminization, hermaphrodites and ambiguous sex. As in our case, Pierga et al. have also described a case of dysgerminoma in a pure 45 , XO Turner syndrome².

It is bilateral in $10 \%$ of cases. Dysgerminoma complicating pregnancy, though rare, has been reported in 60 cases in literature worldwide. Ultrasound is the first investigation to get the clue. According to Mainz et al, 10 sonographic parameters are assessed and scored on a scale of $0-2$, a score of less than 9 is rated as benign; the sensitivity of this scoring is $96.3 \%$. The conventional USG is not diagnostic. CT scan is the most common imaging modality used to stage ovarian tumours; MRI has been shown to be equally acccurate 4 . Many germ cell tumours produce biochemical markers like Beta HCG, Alfa Fetoprotein which help in monitoring the response to treatment. Dysgerminoma is commonly devoid of hormonal product.

Small percentage of tumours produces low levels of HCG. LDH is frequently seen in a patient of dysgerminoma. CA-125 level, though elevated, is not specific. FNAC is not recommended due to the risk of dissemination 5 . The sensitivity is only $25 \%$, specificity being $90 \%$. Metastatic spread is mostly via the lymphatic system, does not occur early in the course of 
disease, and because of that a 5-year survival is 75-90\%. Unfavourable prognostic parameters include presence of metastases at the time of diagnosis, presence of adhesions and spread into adjacent structures, presence of bilateral tumour and presence of other neoplastic germ cell elements. Surgical staging is essential for determining the extent of disease.

In Dysgerminoma stage 1A, unilateral salpingo-oophorectomy with proper follow-up is usually sufficient6. The 10 year survival rate following conservative surgery is $88.6 \%$. Uneventful pregnancy following conservative surgery is also reported ${ }^{1}$. About $15-20 \%$ of dysgerminoma recurs but can be successfully treated with combined chemotherapy at the time of recurrence with a high likelihood of cure. This is one of the success 10 years back diagnostic laparotomy was done, with removal of mass, unilateral salpingo-oophorectomy conserving the uterus and opposite ovary, as suggested for the dysgerminoma of stage 1 without any ascites. Then patient was lost for follow up. 10 years later she came back with mass per abdomen. On high index of suspicion and proper investigations a probable diagnosis of dysgerminoma of the contralateral ovary was made. Staging laparotomy with total abdominal hysterectomy with oophorectomy was done. Histopathology report showed DYSGERMINOMA -STAGE 1A. She was again lost for follow up because of financial constraints.

CONCLUSION: The case is interesting because of its heterogenous behaviour.

\section{REFERENCES:}

1. S. K. Fotiou, Ovarian Malignancies in Adolescence; Annals of the New York Academy of Sciences, , New York Academy of Sciences; 816: 1; 1997: 338-346.

2. Pierga JY, Giacchetti S, Vilain E, Extra JM, Brice P, Espie M, et al.Dysgerminoma in a pure 45, X0 Turner syndrome: report of a case and review of literature. Gynecol Oncol 1994;55:459-64.

3. Mainz e, weber g, Bahaman f, et al. a new sonographic scoring system (Mainz score) for the assessment of tumors using TVS.ultraschaill med 1998;19(3):99-107

4. Woodward PJ, Hossinzadeh K, Saengaer JS, From the archives of the AFIP: Radiologic Stagingof Ovarian Carcinoma with Pathologic Rorrelation. Radiographics 2004 jan-Feb; 24(1):225-46.

5. Higgins R V,Matkins J F, Marroum M C. comparison of FNAC findings of ovarian cysts with histological findings.Am J Obstet Gynaecol 1999;180(3):550-3.

6. Thomas GM, Dembo AJ, Hacker NF, et al. Current therapy of dysgerminoma of the ovary, Obstet gynaecology 1987;70(2):268-75. 


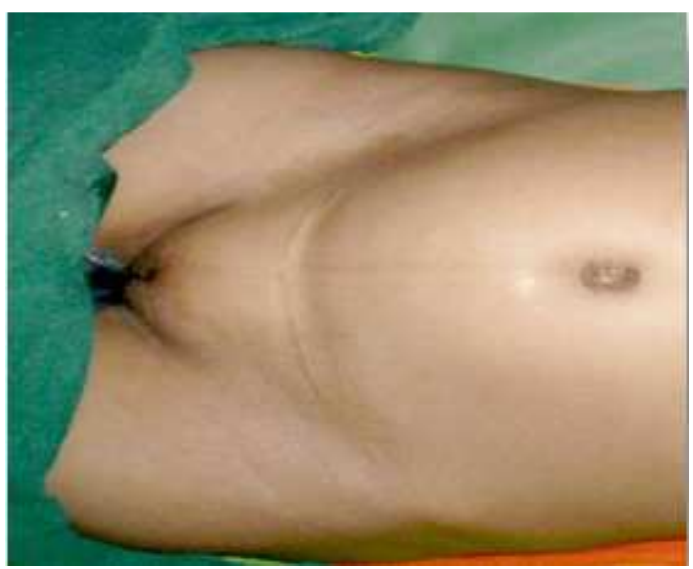

Figure-1

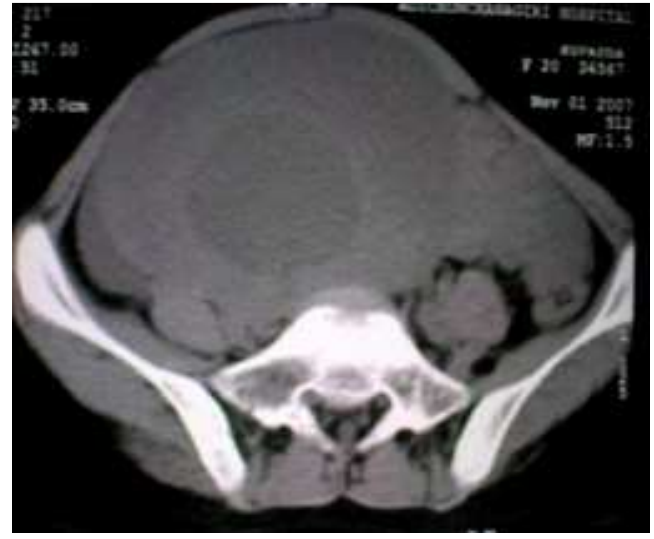

Figure-2

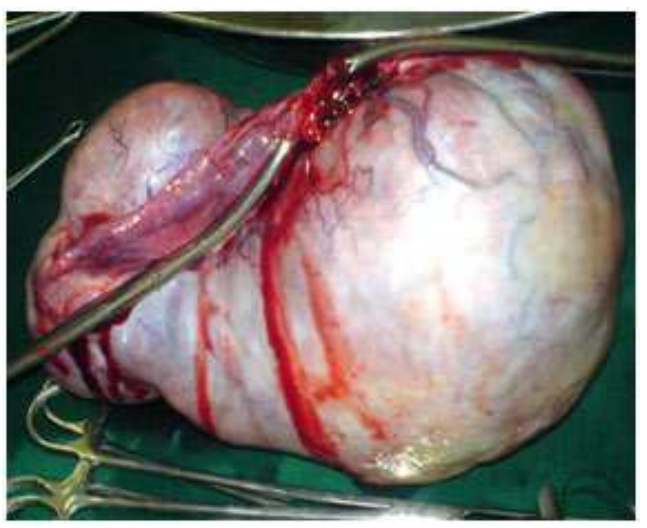

Figure-4

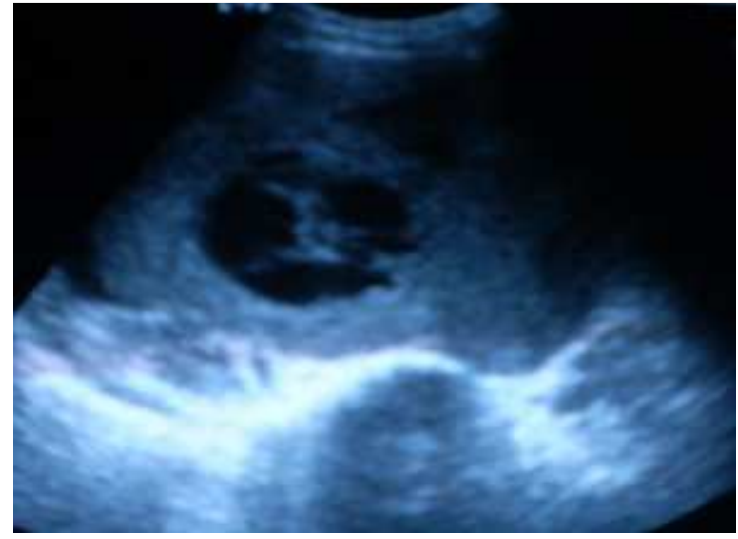

Figure-3

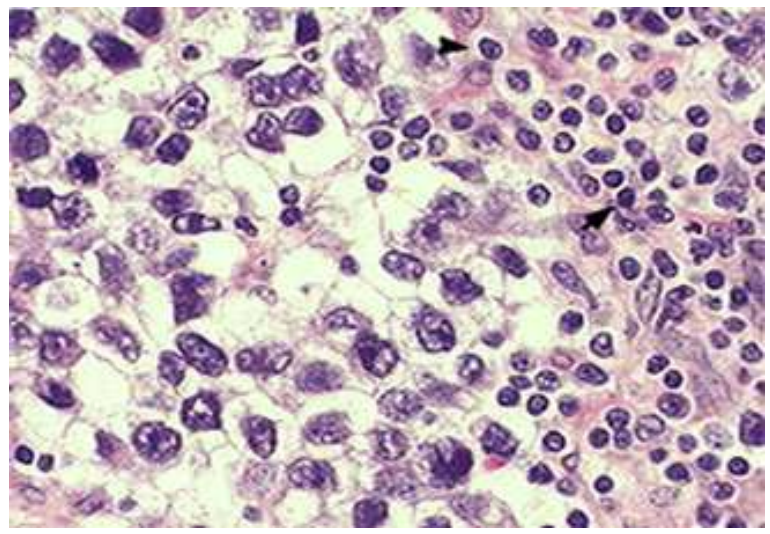

Figure-5 